Perffl de

personalidad en

consumidores

de sustancias

psicoactivas.

Gutiérrez, Hernández y Membreño. 


\section{Perfil de personalidad en consumidores de sustancias psicoactivas.}

Personality profile of psychoactive substances consumers
JoséLuis Gutiérrez

Psicólogo, consultor Júnior en recursos humanos y estrategia organizacional. https://orcid.org/0000-0003-4475-7542 maryelkarolina1992@gmail.com

Eveling Hernández López.

Psicóloga, promotora Psicosocial en el área de jóvenes en conducta de riesgo. https://orcid.org/0000-0001-6978-7911 evelinghdz95@hotmail.com

Allison Membreño.

Psicóloga, experiencia en investigación (cualitativa y cuantitativa) en el área clínica, psicosocial y educativa

https://orcid.org/0000-0002-9524-9911 alicaceres07@gmail.com

DOI: https://doi.org/10.5377/hcs.v0i14.9715

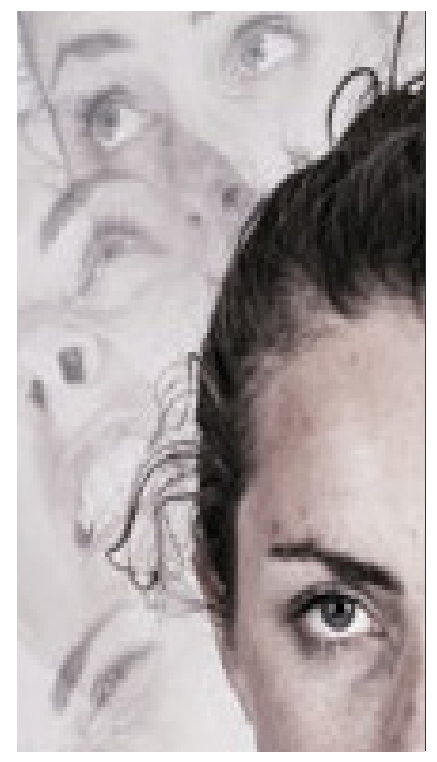

Recibido: 16 agosto 2019

Aceptado: 10 octubre 2019

\section{RESUMEN}

El objetivo principal de este estudio fue comparar el perfil de personalidad de usuarios de centros de rehabilitación en adicciones de Managua (Grupo A) y estudiantes de Psicología de la UNAN-Managua (Grupo B). Se identificó la sustancia de impacto, los niveles de riesgo y se describieron los perfiles por cada sustancia de impacto de los usuarios. La investigación fue de enfoque cuantitativo, de diseño no experimental, corte transversal y alcance descriptivo-comparativo. Se evaluaron un total de 82 personas, 41 casos del Grupo A y 41 casos del Grupo B. Se aplicó "La prueba de detección de alcohol, tabaco y otras sustancias ASSIST-3" y el "Inventario Multifásico de la Personalidad de Minnesota MMPI-2". Para el procesamiento de datos se utilizó el programa IBM SPSSv.22. y para el análisis, estadística descriptiva-comparativa. Se concluyó que existe diferencia significativa entre el perfil de personas del Grupo A y el Grupo B; los usuarios presentaron puntaciones superiores al promedio en las escalas: incoherencia $(F)$, desviación psicopática $(\mathrm{Pd})$, paranoia $(\mathrm{Pa})$, hipomanía $(\mathrm{Ma})$ y esquizofrenia $(\mathrm{Es})$, en contraste con el perfil de estudiantes el cual se ubica dentro de la media, se considera normal y esperable.

\section{ABSTRACT}

The main objective of this research was to compare the personality profiles of users from addiction rehabilitation centers of Managua (group A) and psychology's students from UNAN-Managua (group B) for its subsequent comparison. Equally, identify the substance of impact, the risks levels, and to describe profiles for each substance of impact from the users. The research was of quantitative approach, of non-experimental design, transversal cut and of descriptive-comparative scope. 82 people were evaluated, 41 cases from group $A$ and 41 cases from group $B$. The tests "Alcohol, smoking and substance involvement screening test ASSIST-3" and "Minnesota Multiphasic

\section{PALABRAS CLAVE}

Características de personalidad, sustancias psicoactivas, consumo, estudiantes, comparación

\section{KEYWORDS}

Personality characteristics, psychoactive substances, consumption, students, comparison 
Personality Inventory-2 MMPI-2" were applied. For data processing the program IBM SPSSv.22 was used. And for the analysis, descriptive-comparative statistics. It was concluded that there is significant difference between profiles of people from Group A and Group B, the users presented scores above average in the scales: incoherence $(\mathrm{F})$, psychopathic deviation $(\mathrm{Pd})$, paranoias $(\mathrm{Pa})$, Hypomania (Ma) and schizophrenia (Es), in contrast to the profile of students which is within average, it is considered normal and expected.

\section{Introducción}

La presente investigación Perfil de personalidad y consumo de sustancias psicoactivas en usuarios de Centros de Rehabilitación en adicciones de Managua y estudiantes de Psicología de la UNAN-Managua durante el período febrero-mayo, 2019, este estudio lleva por objetivo comparar el perfil de personalidad de usuarios de centros de rehabilitación en adicciones de Managua (Grupo A) y estudiantes de Psicología de la UNAN-Managua (Grupo B). Para ello se identificaron las sustancias psicoactivas de impacto y el nivel de riesgo en los usuarios, así mismo se describió el perfil de personalidad de estos y se determinó el perfil según el tipo de sustancia de impacto. También, se describió la personalidad de los estudiantes de Psicología de la UNAN-Managua.

Como hipótesis, se plantea que hay diferencia significativa entre el perfil de personalidad de usuarios de centros de rehabilitación y el de estudiantes de psicología.

El abuso de las sustancias psicoactivas y su repercusión en la población, es uno de los fenómenos que recibe considerable atención a nivel mundial en cuanto a salud pública se refiere, lo cual no difiere del contexto nicaragüense; Las investigaciones psicológicas en torno al tema, se basan usualmente en factores sociodemográficos (Tirado, Álvarez, Velásquez y Gómez, 2009), asociación del tratamiento con alguna comorbilidad (Becoña, Casete, Lage y Díaz, 2007) o rasgos de personalidad (López ( Miranda y Gutiérrez, 2005) y en el caso de este estudio, se ha tomado como eje a la personalidad. Esta decisión se debe a que puede ser vista como una de las claves para entender la relación entre el individuo que consume y la sustancia como tal (Pedrero y Rojo, 2008).

\section{Metodología}

El presente estudio es de enfoque cuantitativo, de diseño no experimental, de corte transversal (febrero-mayo 2019) y de alcance descriptivo comparativo.

Muestra: La investigación presenta dos grupos objetivo, los cuales poseen características sociodemográficas similares como nivel académico universitario, rango etario de 19 a 30 años y procedencia de Managua. El Grupo A compuesto por usuarios de centros de rehabilitación de Managua; Centro de Especialidades en Adicciones (CEA), Centro de Ayuda y Rehabilitación del Alcohólico y Adicto a otras drogas (CARA) y Centro Nicaragüense de Solidaridad (CENICSOL). Y el Grupo B, estudiantes de primero a quinto año de la carrera de psicología de la Universidad Nacional Autónoma de Nicaragua, Managua. La población estuvo compuesta por 56 individuos del Grupo A y 500 individuos del grupo B, donde la muestra tomó a 41 personas de cada grupo respectivamente, con un total de 82 participantes.

El muestreo fue no probabilístico en base a criterios de inclusión y exclusión, por lo que para la admisión de personas en el Grupo A, se establece que: deben ser usuarios inscritos en los centros CEA, CARA o CENICSOL, contar con diecinueve años de edad o una edad superior, estar en condiciones permisibles para su colaboración y tener disposición a participar. Por otra parte, para pertenecer al Grupo B: deben ser estudiantes matriculados en la carrera de psicología de la 
UNAN-Managua, contar con diecinueve años de edad o una edad superior, tener disposición para participar y presentar un consumo bajo o nulo de cualquier sustancia psicoactiva (puntajes iguales o menores de tres puntos en cada una de las sustancias descritas por el ASSIST3).

Instrumentos: Para esta investigación se utilizaron dos instrumentos estandarizados. El Inventario Multifásico de Personalidad de Minnesotta 2 (MMPI-2. Minnesota Multiphasic Personality Inventory-2 ()) desarrollado por James Butcher y un equipo de colaboradores. Es una prueba estandarizada que explora las características de personalidad, posibles desviaciones psicopatológicas y trastornos somatoformes. Se utilizaron las escalas clínicas: Histeria (Hi), Depresión (D), Paranoia (Pa), Esquizofrenia (Es), Psicastenia (Pt), Desviación Psicopática (Dp), Hipocondriasis (Hs), Hipomanía (Ma) e Introversión social (Is); y tres escalas de validación que son $\mathrm{L}$ y $\mathrm{F}$ (como escalas de fiabilidad) y $\mathrm{K}$ (factor $\mathrm{K}$ de corrección).

La prueba de detección de alcohol, tabaco y otras sustancias 3 (The Alcohol, Smoking and Substance Involvement Screening Test ASSIST3) desarrollado por un equipo multidisciplinario de la OMS. EI ASSIST3 es una entrevista estructurada de ocho preguntas con respuestas específicas y cerradas sobre la frecuencia del consumo, las formas y las sustancias consumidas durante toda la vida y especialmente los últimos tres meses. Este instrumento permite describir el tipo de consumo de personas en atención primaria, específicamente los niveles de riesgo y necesidad de intervención en rango bajo, moderado y alto.

Procedimiento: El proceso de recolección de la información comenzó con la búsqueda de participantes voluntarios para la investigación tanto en el Grupo A, por medio del contacto con los centros; como en el Grupo B, con una convocatoria en base a los criterios de inclusión y exclusión del estudio. Con ello, se aplicaron los dos instrumentos establecidos (MMPI-2 y protocolo de ASSIST3), para luego corregir su validez y utilidad según las normas y características necesarias para el estudio. A continuación, se codificaron los datos en tres bases del Paquete Estadístico de Ciencias Sociales (Statical Package for the Social Sciences, SPSS versión 22), una base para el grupo A, otra para el grupo $\mathrm{B}$ y la última para la comparación de ambos grupos, con el uso estadística descriptiva para el análisis de los datos.

Se utilizó tablas de distribución de frecuencias para identificar las sustancias de impacto y los niveles de riesgo para las personas del Grupo A únicamente, ya que a ellos se les consideró para tal caso por ser consumidores. En cuanto a la descripción de perfiles de personalidad del Grupo A, Grupo B y según sustancias de impacto, se decidió utilizar la media estadística (medida de tendencia central). Y por último para la comparación de valores de los perfiles de ambos grupos se utilizó la Prueba t de Student para muestras independientes; la cual exploró diferencias entre las medias de cada escala del MMPI-2 con un nivel de significancia $\otimes=0.05$.

\section{Resultados}

En este segmento se presentan los resultados obtenidos de la aplicación de los instrumentos utilizados. Se muestra el análisis a través de la distribución de frecuencias, media estadística, comparación gráfica y estadística (de la cual solo se presentarán los datos significativos) procesadas por medio de SPSS.

Al hablar de sustancia de impacto, esto no implica que los usuarios solo consuman tal sustancia, puesto que pueden consumir más de dos sustancias, sino que es aquella que presenta el mayor nivel de riesgo y necesidad de intervención.
Sustancias de impacto

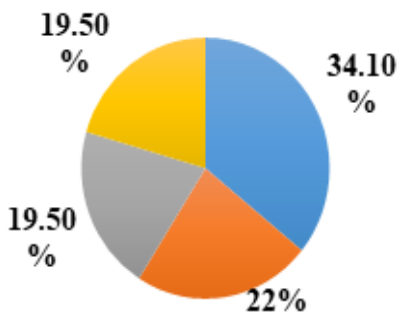

- Tabaco $\square$ Alcohol

- Cannabis $₫$ Cocaina 
El ASSIST3 establece que toda sustancia en que el usuario presenta un nivel de riesgo moderado, este puede tener problemas de salud en dependencia de la sustancia de consumo, por ejemplo, el tabaco ocasiona enfermedades pulmonares, el alcohol provoca afectaciones en el hígado y el corazón, la cocaína, por otra parte, desarrolla problemas intestinales y enfermedades degenerativas. Así mismo, un nivel de riesgo moderado implica una mayor predisposición a que el sujeto desarrolle un trastorno por consumo y dependencia. En el caso del cannabis, que presenta un nivel de riesgo bajo, es menos probable que se desarrolle efectos físicos y hay una menor predisposición a desarrollar un trastorno por consumo.

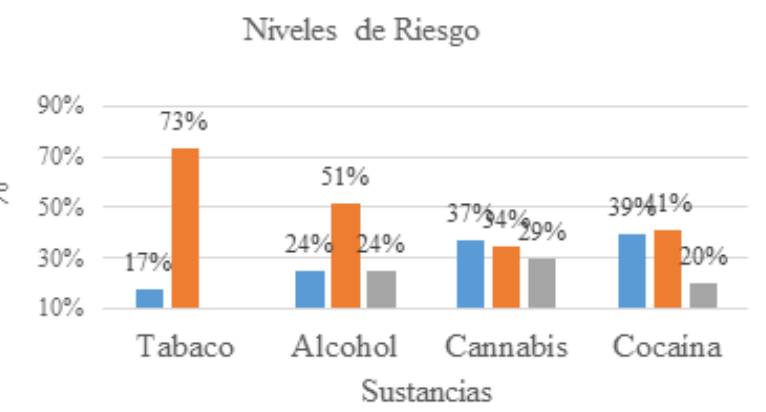

" Nivel Bajo $\quad$ Nivel Moderado $\quad$ Nivel Alto

Respecto al perfil de personalidad del Grupo A, se describen aquellas escalas que presentan un puntaje por encima del promedio establecido por el MMPI-2. Estas escalas son las siguientes:

Perfil de personalidad de usuarios de centros de rehabilitación (CEA, CARA, CENICSOL).

Total de casos: 41 .

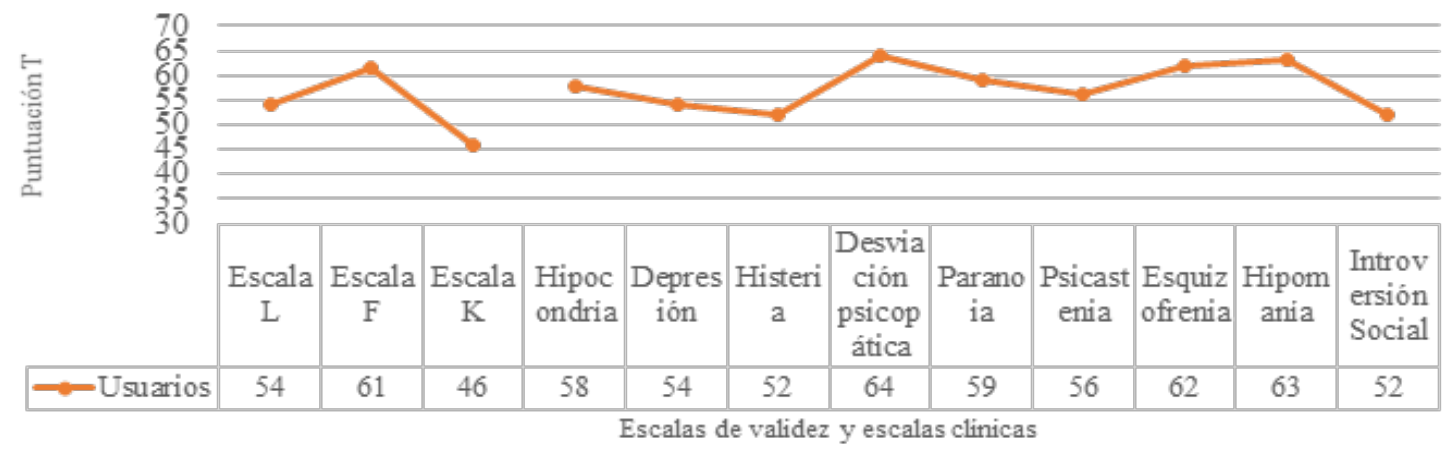

Incoherencia $(\mathbf{F}, \mathbf{T}=\mathbf{6 1})$ refleja un adecuado funcionamiento de los usuarios, pero poseen problemáticas en áreas como el trabajo, sexo, salud u otro aspecto particular. Por otra parte, en Desviación psicopática $(\mathbf{T}=\mathbf{6 4})$ se presentan como personas inmaduras y narcisistas, con problemas ante la ley y es muy probable que exista un consumo de drogas no autorizadas. Experimentan problemas con las personas que le rodean debido a que, si bien muestran ser simpáticos, esto solo es una forma de manipular a las personas y también se les complica el poder establecer una relación profunda con las demás personas.

Paranoia ( $T=59)$ Estas personas se disgustan con facilidad y poseen poca confianza para expresarse, carecen de control emocional y a la vez se les dificulta hablar de ello con otras personas, puesto que tienden a ser esquivos y distantes. Mientras que según Esquizofrenia $(T=62)$, tienen una rutina poco convencional, reflejan un estilo libre esquizoide, se mantienen distantes y se sienten diferentes de los demás. Su juicio puede estar deficiente y en ocasiones podrían perderse dentro de sus fantasías y enfocarse en sus ensueños. Hipomanía $(T=63)$ Los usuarios suelen ser muy activos y energéticos, teniendo un amplio rango de intereses. Pero esa energía lleva a verterse de forma inadecuada, llegando a ser impulsivos, correr grandes riesgos y con altas posibilidades de desarrollar abusos de sustancia, principalmente con las bebidas alcohólicas. 
Perfiles de personalidad por sustancias de impacto

(Estimulantes)

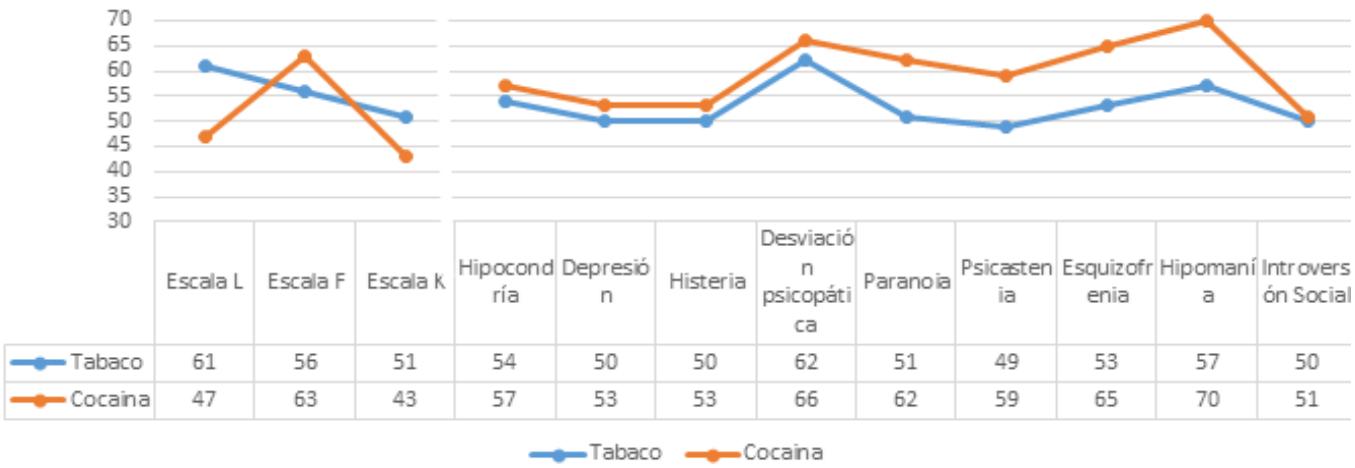

En el perfil por consumo de tabaco, se visualiza que, en contraste con los demás perfiles, este contiene menos escalas por encima del promedio establecido por el MMPI-2, la escala L Mentira, la cual describe personas con actitud defensiva y la escala de Desviación psicopática. En el perfil por consumo de cocaína los puntajes por encima del promedio son las escalas $\mathrm{F}$ Incoherencia, $\mathrm{K}$ Corrección y así mismo las escalas de Desviación psicopática, escala de paranoia e hipomanía lo que implica que son hipersensibles, irritables y en algunos casos agresivos.

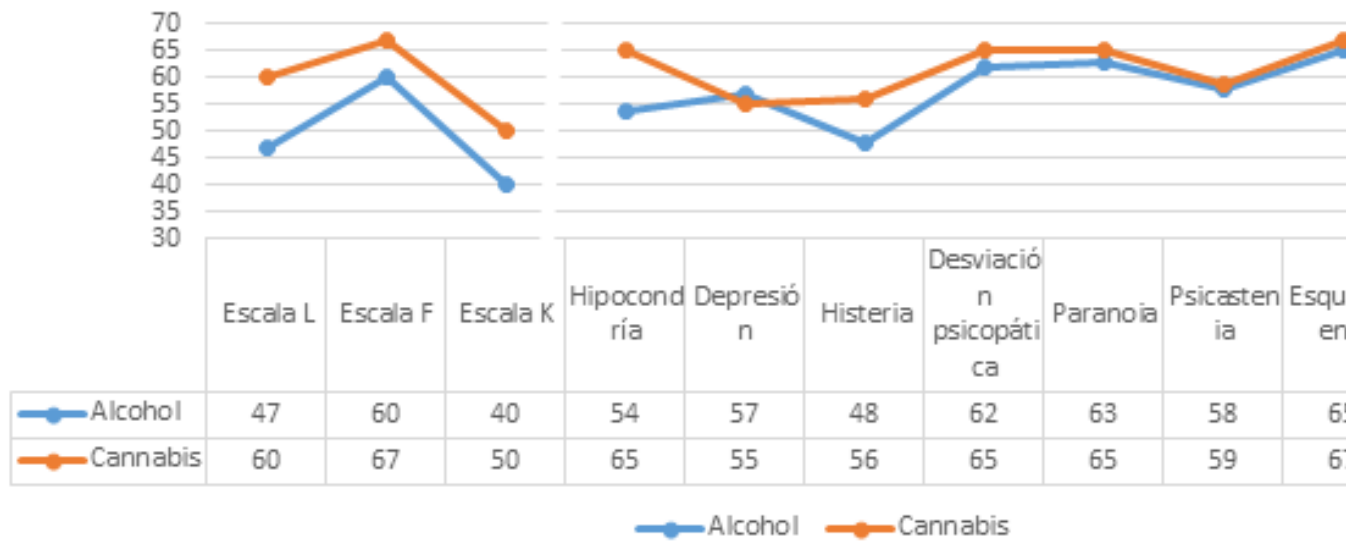

Con respecto al perfil por consumo de alcohol, se presentan puntajes por encima del promedio en las escalas $L$ mentira, $F$ incoherencia, $K$ corrección, desviación psicopática, paranoia, esquizofrenia e hipomanía, por lo que se les puede describir como personas con poca tolerancia a la frustración, juicio deficiente, e hipersensibles. Por otra parte, el perfil por consumo de cannabis, indica puntajes por encima del promedio en las escalas $L$ mentira, $F$ incoherencia, Hipocondría, desviación psicopática, paranoia, esquizofrenia e hipomanía describiendo a personas emocionalmente inestables, con dificultades para relacionarse con los demás, poco juicio e impulsividad. De igual forma presentan bastantes preocupaciones somáticas.

La gráfica de comparación de ambos perfiles A y B, muestra cinco escalas del perfil de usuarios con puntajes superiores al perfil de estudiantes (los cuales se encontraron dentro del promedio establecido por el MMPI-2), donde desviación psicopática es la más destacable; retomando uno de los antecedentes encontrados en donde también se elaboró un perfil en personas con drogodependencia, la escala de desviación psicopática también presenta un puntaje superior al promedio establecido por el MMPI-2. Por lo tanto, una de las principales diferencias recae en la capacidad de adaptación que ambos grupos tienen con respecto a su medio. 


\section{Discusión}

En los resultados de sustancias de impacto las cuales son las que generan mayores efectos negativos en los usuarios, se identificó que el tabaco y las bebidas alcohólicas ocupan los primeros dos lugares, lo cual coincide con el contexto nacional e internacional, como tal señalan estudios de España, donde su consumo es bastante elevado (Policía Nacional, 2013). Desde la perspectiva del aprendizaje social, la publicidad de estos productos asocia el consumo con una respuesta emocional positiva y provoca altas expectativas sobre los efectos del mismo.

\section{Comparación de Perfiles de Personalidad, Usuarios en Centros de Rehabilitación y \\ Estudiantes de Psicologia de la UNAN-Managua. \\ Cantidad de casos: 41 Usuarios, 41 Estudiantes.}

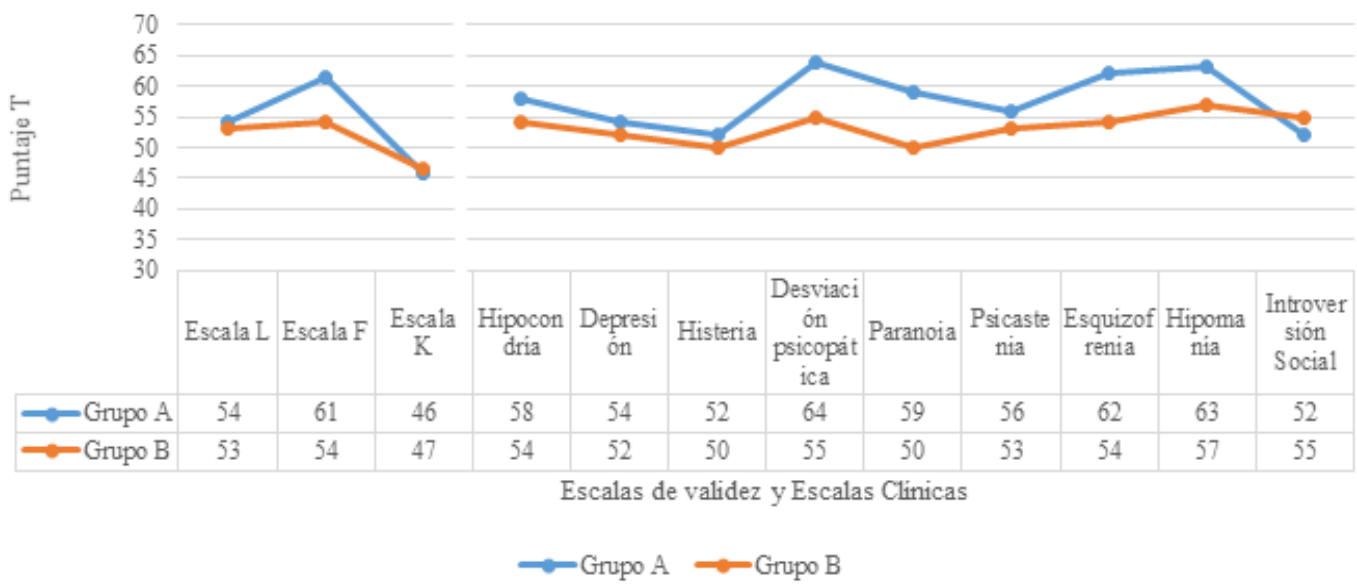

Pese a que la adquisición de ambas sustancias es permitida a partir de la mayoría de edad, en muchos casos se inicia desde la adolescencia e incluso la niñez. En muchos contextos su consumo es reforzado por la interacción social de las personas que rodean al individuo, lo adquiere como propio a través experiencias y ellas le incitan al consumo posteriormente. En la perspectiva conductual, el alcohol y el tabaco actúan como reforzadores positivos que producen sensaciones placenteras y actúan como reforzamiento negativo cuando se produce alivio a un estado displacentero (síndrome de abstinencia), lo cual se transforma en un control conductual bidireccional entre la presencia y ausencia de la sustancia.

Por otro lado, la marihuana y la cocaína son las terceras sustancias de impacto y desde la teoría del modelo biológico, estas sustancias actúan sobre zonas como la vía dopaminérgica mesolímbica, que es la encargada de producir y enviar dopamina a diversos grupos de células nerviosas en partes delanteras del encéfalo, lo cual bloquea la recaptación de la dopamina en las terminales nerviosas y como resultado, aumenta los efectos de la sustancia considerablemente, promoviendo la continuidad del consumo (Organización Mundial de la Salud, 2005).

Según los resultados del perfil de personalidad del grupo A, se evidencia estrecha relación con los criterios de trastornos por consumo de sustancias. Por ejemplo, el criterio de consumo prolongado con aumento en las dosis de las sustancias, se relaciona con la característica de personalidad del grupo A, que indica una alta predisposición a desarrollar abuso de las drogas no autorizadas (American Psychiatric Association, 2014). 
A su vez, su tendencia a desarrollar problemas en áreas tales como la familia, la salud o el trabajo, se asimila a uno de los criterios básicos de los trastornos por consumo de sustancias, en referencia a que el individuo se descompensa abandona sus actividades ocupacionales, recreacionales y sus lazos familiares, para satisfacer la necesidad del consumo (APA, 2014). Otro dato del perfil del grupo $A$, es la posibilidad de una patología dual, a razón de elementos que indican la presencia de otros tipos de trastorno.

La escala desviación psicopática junto con hipomanía, revelaron características asociadas con el trastorno de la personalidad antisocial, puesto que, en sus criterios, se resalta el incumplimiento de las normas sociales, estafas para provecho personal, impulsividad, irritabilidad, falta de una conducta laboral adecuada y al menos tener como mínimo 18 años de edad (APA, 2014). Por lo que, con los criterios antes mencionados, es posible relacionar las características encontradas en el grupo A con dicho trastorno; esto en concordancia con la edad de los miembros de la muestra, su alta predisposición a comportamientos que entren en conflictos con las leyes establecidas con la sociedad y la tendencia a irritarse o actuar energéticamente sin considerar las consecuencias de sus actos.

Sin embargo, la APA (2014) enfatiza que, para diagnóstico de una patología dual, cuando existe un trastorno por consumo de sustancias, es imprescindible que el trastorno de personalidad antisocial también indique precedentes de haber iniciado desde la infancia. Con lo antes mencionado estas características de personalidad pueden tomarse como factores de riesgo para que un individuo desarrolle dependencia a sustancias, pero, por otra parte, existe la posibilidad de que el consumo de determinadas sustancias exacerbe ciertos rasgos de personalidad encontrados en el grupo A; ambos aspectos pueden ser abordados a través de los resultados de los perfiles por sustancias de impacto.

Respecto al objetivo de los perfiles de personalidad por sustancias de impacto de encontró que comparten la tendencia a conductas contrarias con las normas sociales y a desarrollar una dependencia por consumo, pero en sus diferencias, los consumidores de tabaco, reflejan ser el perfil con menos rasgos alterados, y es por ello que se presume mantienen a esta sustancia como la principal; debido a que el tabaco es un estimulante menor y sus efectos a corto y a largo plazo no inciden en conductas que interfieran con las normas sociales como lo hacen otras sustancias.

Por otra parte, en el perfil alcohol, sus rasgos les describen como personas que no asumen sus propias responsabilidades, son inseguras y emocionalmente inestables, por lo que se presume eligen a esta sustancia como medio de escape o desahogo, a razón de que el alcohol disminuye las funciones cognitivas, perceptivas y motoras, lo que obstaculiza el pensamiento coherente y lleva a tener dificultades con la memoria llegando a la amnesia (APA, 2014).

Respecto al perfil de cannabis, se encuentra que tienen muchas preocupaciones sobre dolencias o malestares, lo que podría respaldar su preferencia a dicha sustancia. Debido a que es sujeta a variados debates a nivel mundial, por ser considerada una panacea para diversas dolencias, afectaciones crónicas o malestares generales (National Institute on Drug Abuse, 2019). Por otra parte, como se sugirió anteriormente, puede suceder que algunas características estén directamente ligadas a la constante exposición a ciertas sustancias, como el cannabis o la cocaína, que con el tiempo son capaces de incidir en la atenuación de ciertas conductas y reacciones. 
Cannabis y cocaína comparten una notoria elevación en la escala manía. Lo cual se refiere a irritabilidad o agresividad y energía mal dirigida; característica que podrían haber sido estimuladas o exacerbados por los efectos de ambas sustancias en el sistema nervioso y su incidencia en la conducta manifiesta.

En referencia a los resultados del perfil de personalidad del grupo $\mathrm{B}$, se reflejaron puntajes dentro de la media establecida por el MMPI-2. Lo cual luce acorde a la formación profesional del grupo, donde se muestran con un adecuado nivel de adaptabilidad, congruencia con el entorno al que pertenecen y son capaces de desempeñarse apropiadamente. Los resultados de la comparación del perfil del grupo A y grupo B permiten identificar una marcada diferencia, la cual, puede tener como base a los mecanismos de acción y sus efectos en los miembros de cada grupo, así como los cambios continuos del ambiente con los que ambos grupos interactúan. $Y$ si bien pudieron estar expuestos al mismo contexto de crecimiento, es posible que el grupo $B$ se haya expuesto a elementos que se esfuerzan en orientarles profesionalmente a pesar de la presencia de propagandas o estímulos de consumo.

\section{Conclusiones}

Se procede a concluir que existe diferencia significativa en la comparación estadística entre ambos perfiles donde el Grupo A obtuvo puntajes más altos frente al Grupo B que se ubicó en la media. La principal sustancia de impacto en el Grupo A (Usuarios de centros de rehabilitación) es el tabaco, con un nivel riesgo moderado, seguido por el alcohol con nivel de riesgo moderado. El perfil de personalidad del Grupo A, los puntajes de esas escalas se ubicaron fuera de la media establecida por el MMPI-2, lo que señala dificultades en el control de sus emociones, conflictos en la formación lazos de afectivos, tendencia a no seguir las normas sociales y a desarrollar dependencia a sustancias no autorizadas. Los perfiles por sustancias de impacto del Grupo A determinan que, en el perfil de personalidad por tabaco, ellos son más confiados en sí mismos, en el perfil alcohol son excesivamente desconfiados con los demás, en el perfil cannabis se presenta predisposición paranoide y susceptibilidad a las somatizaciones, y en el perfil cocaína (crack) suelen detonar en episodios agresivos. El perfil de personalidad del Grupo B los puntajes se ubicaron dentro de la media del MMPI-2, el cual indica que son personas responsables, sociables y con buena capacidad de adaptación.

\section{Referencias bibliográficas}

American Psychiatric Association (APA). (2014). Manual Diagnóstico y Estadístico de los Trastornos Mentales (DSM-5®). México, D.F., México: Editorial Médica Panamericana.

Butcher, J., Graham, J., Ben-Porath, Y., Tellegen, A., Dahlstrom, W. y Kaemmer, B. (2019). MMPI2. Inventario Multifásico de Personalidad de Minnesota-2 ( $4^{\mathrm{a}}$ Ed.) (A. Ávila-Espada y F. Jiménez-Gómez, adaptadores). Madrid: TEA Ediciones.

Cloninger, S. (2003). Teorías de la personalidad. México. PEARSON EDUCACIÓN.

Consejería para la igualdad y Bienestar social y Dirección General para las Drogodependencias. (2006). Curso básico de información / formación sobre drogodependencias y adicciones. 
Dirección General para las Drogodependencias y adicciones (2006) Drogodependencias y Adicciones. Andalucía, España: Junta de Andalucía.

Fernández, M. (2002). Alto consumo de drogas en Nicaragua. La Prensa. Recuperado de https:// www.laprensa.com.ni/2002/06/26/nacionales/876584-alto-consumo-de-drogas-en-nicaragua

González, A. y Matute, E. (2013). Cerebro y drogas. México D.F. México: Editorial Manual Moderno.

Hernández, R., Fernández, C., \& Baptista, P. (2014). Metodología de la investigación: Roberto Hernández Sampieri, Carlos Fernández Collado y Pilar Baptista Lucio (6a. ed.). México D.F., McGraw-Hill.

MedlinePlus (2018) Riesgos del consumo de Alcohol para la salud. Biblioteca nacional de medicina de Estados Unidos. Recuperado de: https://medlineplus.gov/spanish/ency/patientinstructions/000494.htm

MedlinePlus (2018) Riesgos del Tabaco. Biblioteca nacional de medicina de Estados Unidos. Recuperado de: https://medlineplus.gov/spanish/ency/article/002032.htm

National Institute on drug abuse (NIDA) (2019) La marihuana como medicina. Recuperado de https://www.drugabuse.gov/es/publicaciones/drugfacts/la-marihuana-como-medicina

Organización Mundial de la Salud (OMS) (2018). Informe Mundial sobre las Drogas 2018. Recuperado de https://www.unodc.org/wdr2018/prelaunch/WDR18_ExSum_Spanish.pdf

Organización Mundial de la Salud (OMS) (2005). Neurociencia del consumo y Dependencia de Sustancias Psicoactivas. Whashintong D.C., Estados Unidos.

López, A., Becoña, E., Casete, L., Lage, M., Díaz, E., García, J., Senra, A., Cancelo, J., Estévez, C., Sobradelo, J., Vieitez, I., Lloves, M. y Moneo, A. (2007). Dependencia de la cocaína y trastornos de personalidad. Análisis de su relación en una muestra clínica. Trastornos Adictivos. Recuperado de https://www.elsevier.es/es-revista-trastornos-adictivos-182-articulo-dependencia-cocaina-trastornos-personalidad-analisis-13109737

Tirado, A., Álvarez, M., Velásquez, J., Gómez L., Ramírez C. y Vargas, A. (2009). Prevalencia y factores de riesgo para el consumo y dependencia de drogas en estudiantes de una universidad de Medellín, Colombia, 2009. Revista Facultad Nacional de Salud Pública. Recuperado de http:// aprendeenlinea.udea.edu.co/revistas/index.php/fnsp/article/view/9682/10909 


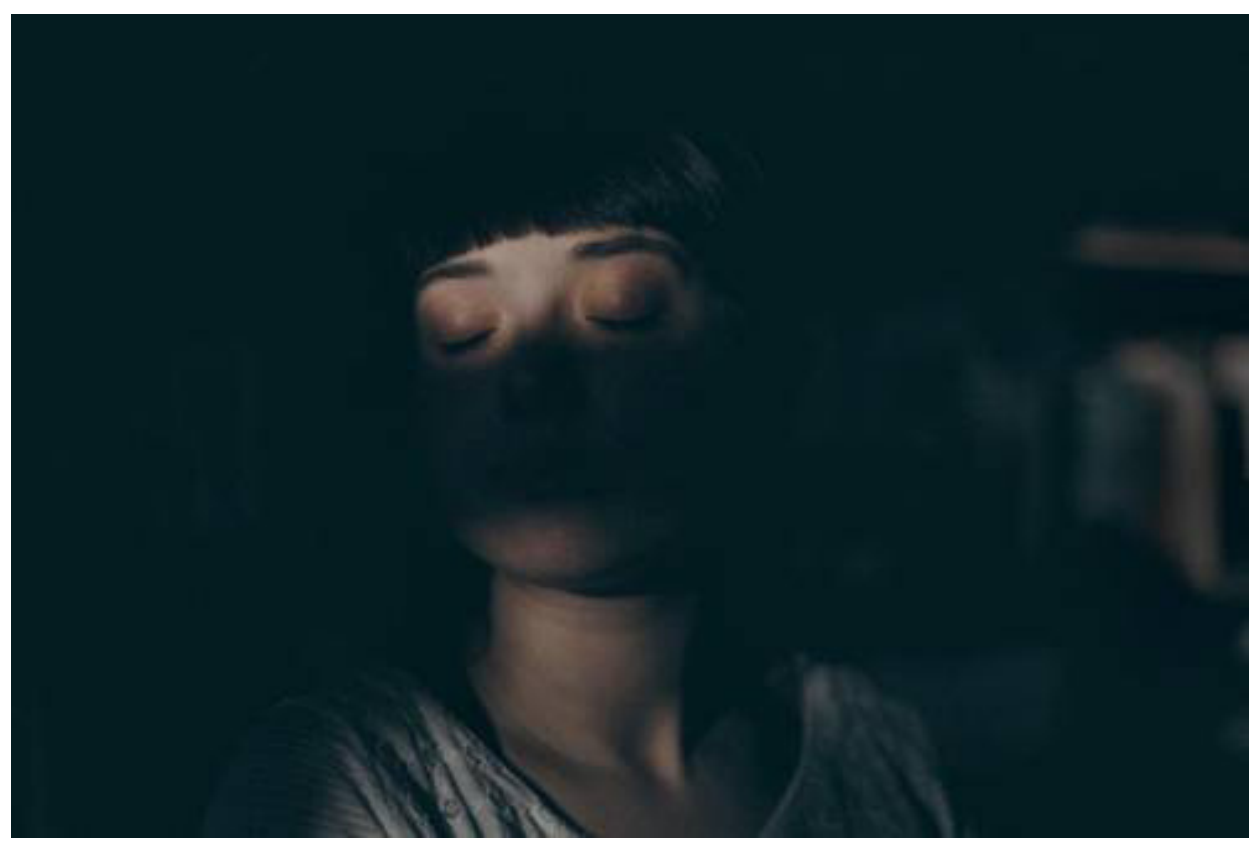

José Luis Gutiérrez

Psicólogo. Consultor Júnior en recursos humanos y

estrategia organizacional.

https://orcid.0000-0002-3951-3108

maryelkarolina1992@gmail.com

Eveling Hernández López.

Psicóloga. Promotora Psicosocial en el área de jóvenes en conducta de riesgo.

https://orcid.ORCID: 0000-0001-6978-7911

evelinghdz95@hotmail.com

Allison Membreño. Psicóloga.

- Psicóloga. Experiencia en investigación (cualitativa

y cuantitativa) en el área clínica, psicosocial y

educativa

https://orcid.0000-0002-9524-9911

alicaceres07@gmail.com 